\title{
Classical Spin Systems in the Presence of a Wall: Multicomponent Spins
}

\author{
Jürg Fröhlich ${ }^{1}$ and Charles-Edouard Pfister ${ }^{2}$
}

1 Theoretische Physik, ETH-Hönggerberg, CH-8093 Zürich, Switzerland

${ }^{2}$ Départements de mathématiques et de physique E.P.F.-L., CH-1015 Lausanne, Switzerland

\begin{abstract}
In this paper we investigate classical spin systems on a semi-infinite lattice. We establish detailed properties of such systems near the surface layer. For the Ising- and the classical $X Y$ models on a semi-infinite lattice we study the phase diagram, the critical properties and the decay of spin-spin correlations near the surface layer.
\end{abstract}

\section{Introduction}

\subsection{The Models}

This paper is devoted to the study of some surface problems for classical bounded spin systems with a continuous internal symmetry group $G$. We consider models on a semi-infinite sublattice $\mathbb{L}$ of $\mathbb{Z}^{3}$, say $\mathbb{L}=\left\{x=\left(x^{1}, x^{2}, x^{3}\right) \in \mathbb{Z}^{3}: x^{3} \geqq 0\right\}$. We propose to study the behaviour of the system near the boundary surface $\sum$, $\Sigma=\left\{x \in \mathbb{L} ; x^{3}=0\right\}$. Let us introduce the simplest model of this kind. The spin at $x \in \mathbb{L}$ is described by a unit vector in $\mathbb{R}^{n}, S(x)=\left(S^{1}(x), \ldots, S^{n}(x)\right), n \geqq 1$, and the Hamiltonian is

$$
-\sum_{\{x, y\}} K(x, y) S(x) \cdot S(y),
$$

where $S(x) \cdot S(y)$ is the Euclidean scalar product in $\mathbb{R}^{n}$. We consider only shortrange interactions and, for the sake of simplicity, we take $K(x, y)=0$ if $x$ and $y$ are not nearest neighbours. If $x$ and $y$ are nearest neighbours,

$$
K(x, y)=K \quad \text { if } \quad\{x, y\} \leftarrow \Sigma,
$$

and

$$
K(x, y)=J \quad \text { if } \quad\{x, y\} \subset \sum .
$$

The inverse temperature $\beta$ is one, and we investigate the behaviour of the model when $J$ and $K$ are varied, and (primarily) for $n \geqq 2$. 


\subsection{Absence of Symmetry Breaking in the Surface Layer}

Since $\Sigma$ is a two-dimensional sublattice and the internal symmetry group is continuous, a natural question is whether one can break the internal symmetry near or on the boundary $\Sigma$. It is well-known that this is impossible on a strictly two-dimensional space. This is the content of the Mermin-Wagner theorem $[1,2]$. However, in our model the bulk, i.e. the part of the system for values of $x^{3}$, with $x^{3} \rightarrow \infty$, is three-dimensional, and the behaviour on the surface will be different according to whether the bulk is ordered or not. To be more specific, let us consider the ferromagnetic model, $J>0$ and $K>0$. We know that there is a critical coupling $K_{c}^{3}$ such that, for $K>K_{c}^{3}$, the bulk is ordered and, for $K<K_{c}^{3}$, the bulk is disordered. In the latter case we do not expect that the bulk part of the system plays a significant role, and therefore we do not expect to have symmetry breakdown near $\Sigma$. In order to prove this result, we compute an effective hamiltonian for the spins on $\Sigma$, using a high-temperature expansion in the coupling $K$. Provided that this expansion is convergent, i.e. for $K$ small enough, we get an effective hamiltonian with many-body interactions which decay exponentially. A straightforward application of the relative entropy argument [3], shows that there is no symmetry breakdown for this effective hamiltonian, for any value of the coupling $J$. Details are given in Sect. 2, for $n=2$. However it is clear that the proof is valid for quite general models. The basic hypotheses we need are the following:

a) The internal symmetry group $G$ is a compact connected Lie group.

b) The interaction is smooth $\left(C^{2}\right)$ with respect to the action of the group and satisfies some decay properties. For precise hypotheses, see [4, pp. 182, 188].

c) We have a convergent high-temperature expansion for the spins which are not on $\Sigma$.

\subsection{Existence of Ordering on the Surface $\Sigma$}

If we consider the opposite situation for which the bulk is ordered, i.e. $K>K_{c}^{3}$ in our ferromagnetic model, we find a completely different behaviour. Intuitively, we can think that the ordered bulk acts upon the spins on $\Sigma$ like an external magnetic field. Therefore the symmetry on $\Sigma$ should also be broken for any value of $J$. This argument is of course not a proof. In the mean field Potts model (\# of components $q \rightarrow \infty$ ) Lipowsky found a disordered surface state in presence of an ordered bulk state [5]. We can prove the existence of a spontaneous magnetization in the ferromagnetic $X Y$ model $(n=2)$ by the method of [6]. Here $n=2$ is a genuine restriction: we cannot adapt the method of [7], which is based on the infrared bound, since we do not have full translation invariance. Therefore, if $J=K$, and $K>0$ is large enough, the spins on $\Sigma$ are ordered in the $X Y$ model. Let $K^{*}$ be the infimum of the couplings $K$ for which the model with $J=K$ is ordered on $\Sigma$. By correlation inequalities, we extend this result to arbitrary values of $J$ and $K>K^{*}$. These topics are discussed in Sect. 3.

\subsection{Phase Diagram for the Ferromagnetic XY Model}

Let us summarize the above results for the ferromagnetic $X Y$ model, $(J>0, K>0$, $n=2)$. It is convenient to parametrize the spins $S(x)$ by an angle $\phi(x)$ so that

$$
S(x)=(\cos \phi(x), \sin \phi(x)) .
$$


If we add an external magnetic field we can choose its direction so that its contribution to the Hamiltonian is given by

$$
-\sum_{x \in \mathbb{L}} h \cos \phi(x) \text {. }
$$

The corresponding equilibrium state is denoted

$$
\langle\cdot\rangle(J, K ; h) \text { and }\langle\cdot\rangle^{+}(J, K)=\lim _{h \downarrow 0}\langle\cdot\rangle(J, K ; h) .
$$

There is a $K^{*}, K_{c}^{3} \leqq K^{*}<\infty$ such that

$$
K^{*}=\inf \left\{K:\langle\cos \phi(0)\rangle^{+}(K, K)>0\right\}
$$

and a $K_{*}>0$ such that

$$
K_{*}=\sup \left\{K:\langle\cos \phi(0)\rangle^{+}(J, K)=0, \forall J\right\} .
$$

In order to describe the behaviour of the model more precisely, we introduce the boundary susceptibility $\chi_{\Sigma}(J, K)$,

$$
\chi_{\Sigma}(J, K)=\sum_{x \in \Sigma}\langle\cos \phi(0) \cos \phi(x)\rangle^{+}(J, K),
$$

the boundary correlation length $\xi_{\Sigma}(J, K)$,

$$
\left(\xi_{\Sigma}(J, K)\right)^{-1}=\lim _{x^{1} \rightarrow \infty}-\frac{1}{x^{1}} \ln \left\langle\cos \phi(0,0,0) \cos \phi\left(x^{1}, 0,0\right)\right\rangle^{+}(J, K),
$$

and the transverse correlation length $\xi_{\perp}(J, K)$,

$$
\left(\xi_{\perp}(J, K)\right)^{-1}=\lim _{x^{3} \rightarrow \infty}-\frac{1}{x^{3}} \ln \left\langle\cos \phi(0,0,0) \cos \phi\left(0,0, x^{3}\right)\right\rangle^{+}(J, K) .
$$

Let us assume that there is no intermediate phase in the three-dimensional model. In other words, if $K<K_{c}^{3}$ there is no bulk spontaneous magnetization, the bulk correlation length is finite and the bulk susceptibility is finite. Let us also make the very plausible assumption that

$$
K_{*}=K^{*}=K_{c}^{3} .
$$

Under these two assumptions the phase diagram is very simple (see Fig. 1). There are three different regions. Region I corresponds to the high temperature phase

Fig. 1

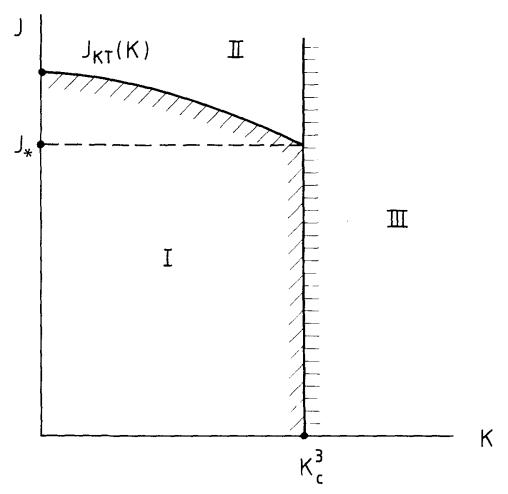


and it is characterized by $\langle\cos \phi(0)\rangle^{+}(J, K)=0$ and $\chi_{\Sigma}(J, K)<\infty$. Region II corresponds to the Kosterlitz-Thouless phase where $\langle\cos \phi(0)\rangle^{+}(J, K)=0$ but $\chi_{\Sigma}(J, K)=\infty$. Region III corresponds to the ordered phase, where $\langle\cos \phi(0)\rangle^{+}(J, K)>0$ and $\chi_{\Sigma}(J, K)=\infty$.

Let us examine region I in more detail. This region certainly contains the square $\left\{(J, K): J<K_{c}^{3}, K<K_{c}^{3}\right\}$. Inside this square the boundary correlation length $\xi_{\Sigma}(J, K)$ is finite, since in the three-dimensional model the bulk correlation length is finite, for $K<K_{c}^{3}$. We can prove, by adapting an argument of Simon [8], that for $K<K_{c}^{3}$

$$
\chi_{\Sigma}(J, K)<\infty \Leftrightarrow \xi_{\Sigma}(J, K)<\infty .
$$

Therefore, inside region $\mathrm{I}, \xi_{\Sigma}(J, K)<\infty$. The boundary of the region consists of two pieces: $\Gamma_{1}$,

$$
\Gamma_{1}=\left\{(J, K): K=K_{c}^{3}, J \leqq J_{*}\right\},
$$

which separates regions I and III, and $\Gamma_{2}$,

$$
\Gamma_{2}=\left\{(J, K): K \leqq K_{c}^{3}, J=J_{K T}(K)\right\},
$$

which separates regions I and II. The function $J_{K T}(K)$ is monotone decreasing in $K, J_{K T}(0)=J_{K T}^{2}$, and $J_{K T}\left(K_{c}^{3}\right)=J_{*} \geqq K_{c}^{3}$. By correlation inequalities

$$
0 \leqq \chi_{\Sigma}^{-1}\left(J_{1}, K\right)-\chi_{\Sigma}^{-1}\left(J_{2}, K\right) \leqq 4\left(J_{2}-J_{1}\right), \quad J_{2} \geqq J_{1} .
$$

Thus $\chi_{\Sigma}^{-1}$ is a continuous function of $J$ and $\chi_{\Sigma}^{-1}(J, K)=0$ on $\Gamma_{2}$ (provided that $\Gamma_{2}$ does not have vertical pieces). $\Gamma_{2}$ is a critical line. In particular, if we go from region I to region II along a straight line $\left\{(J, K): J=\beta J_{0}, K=\beta K_{0}, \beta \geqq 0\right\}$ and $\operatorname{cross} \Gamma_{2}$ at $(\hat{J}, \hat{K}) \equiv\left(\hat{\beta} J_{0}, \hat{\beta} K_{0}\right)$, the susceptibility $\chi_{\Sigma}\left(\beta J_{0}, \beta K_{0}\right)$ diverges at least like $(\hat{\beta}-\beta)^{-1}$. [We have assumed that the function $K \rightarrow J_{K T}(K)$ has a tangent.] At $\hat{\beta}$ we have a Kosterlitz-Thouless transition. If we continue along that straight line, we find a second transition with symmetry breakdown at $\widetilde{\beta}, \widetilde{\beta} K_{0}=K_{c}^{3}$. On the other hand, if $J_{0}$ is small we have only one transition with symmetry breakdown at $\widetilde{\beta}$.

More generally, the inverse correlation length $\xi_{\Sigma}^{-1}(J, K)$ is a continuous function of $J$ and $K$. The proof is the same as in the bulk case, (see e.g. [9]). Inside region I, $\xi_{\Sigma}<\infty$, and when we pass from region I to regions II or III $\xi_{\Sigma}$ diverges. The boundary of region I, $\Gamma_{1} \cup \Gamma_{2}$ is therefore a critical line.

Finally, inside regions I and II we expect that the transverse correlation length is finite. If $J \leqq K$ this correlation length is indeed equal to the bulk correlation length $\xi_{\mathrm{Bulk}}(K)$. However for $J \geqq K$ we have established only the inequality

$$
\xi_{\perp}(J, K) \geqq \xi_{\text {Bulk }}(K) .
$$

It is likely that we have equality in (1.17). This would show that $\xi_{\perp}$ diverges when we go from regions I or II to region III across the line $K=K_{c}^{3}$.

Let us finally remark that we get qualitatively the same phase diagram if we make only the assumption that the model with $J=K$ has no intermediate phase. 


\subsection{Behaviour of the Two-Point Function \\ for Small or Large Values of the Coupling Constants}

In the following, we summarize our results on the behaviour of spin-spin correlations near the surface $\Sigma$. We define

$$
\langle S(x) ; S(y)\rangle \equiv\langle S(x) S(y)\rangle-\langle S(x)\rangle\langle S(y)\rangle,
$$

where the expectation $\langle(\cdot)\rangle$ is given by $\langle(\cdot)\rangle^{+}(J, K)$, and if

$$
S(x)=\left(S^{1}(x), \ldots, S^{n}(x)\right)
$$

is an $n$-component spin $S(x) S(y)$ stands for $\sum_{\alpha=1}^{n} S^{\alpha}(x) S^{\alpha}(y)$.

(1) High-Temperature Behaviour of Spin-Spin Correlations. We assume that the bulk couplings, $K$, and the surface couplings, $J$, are chosen to be so small that the model is well inside the high-temperature phase I. If the external magnetic field vanishes then $\langle S(x)\rangle=0$, i.e. $\langle S(x) ; S(y)\rangle=\langle S(x) S(y)\rangle$ and $\langle S(x) S(y)\rangle$ decays exponentially, as $|x-y| \rightarrow \infty$. It is natural to ask what the power law corrections to exponential decay are. It turns out that this problem is closely related to the problem of the wetting- or unpinning transition for an interface in the twodimensional Ising model studied by Abraham and others $[10,11]$. Mathematically closely related problems are analyzed in $[12,13]$. Using the techniques in $[13]$ one can prove the following

Result. Consider a classical, ferromagnetic $n$-vector model, as described above, in a $d$-dimensional half lattice $\mathbb{L}=\left\{x \in \mathbb{Z}^{d}: x^{d} \geqq 0\right\}$.

(a) If $0<K \ll J \ll 1$, and for $x$ and $y$ in (or near) the surface $\Sigma$,

$$
\langle S(x) S(y)\rangle_{|x-y| \rightarrow \infty} \operatorname{const}|x-y|^{-\frac{d-2}{2}} e^{-|x-y| / \xi}
$$

for some $\xi=\xi_{\Sigma}(J, K)<\infty$.

(b) If $0<J \leqq K \ll K_{c}^{d}$, and for $x$ and $y$ in (or near) $\Sigma$

for some $\xi<\infty$.

$$
\langle S(x) S(y)\rangle_{|x-\tilde{y}| \rightarrow \infty} \operatorname{const}|x-y|^{-\frac{d+1}{2}} e^{-|x-y| / \xi},
$$

The proofs are sketched in Sect. 5 .

Remarks. 1) We shall see that behaviour (a) corresponds to the "dry" phase of the two-dimensional Ising model, while behaviour (b) corresponds to the "wet" phase, [10].

2) Deep inside the bulk [e.g. $x=0, y=(0, \ldots, 0, n)$, with $n \rightarrow \infty)$ one finds the usual Ornstein-Zernike behaviour

$$
\langle S(x) S(y)\rangle_{|x-y| \rightarrow \infty} \text { const }|x-y|^{-\frac{d-1}{2}} e^{-|x-y| / \xi},
$$

where $\xi=\xi_{\perp}(J, K)<\infty$ if $K \ll K_{c}^{d}$. See e.g. [13] for proofs and references to earlier work.

3) Results like those described in (1.18) and (1.19) were discussed, on a heuristic level, by Fisher and Camp [14]; see e.g. the beautiful review of Fisher [11] and refs. 
given there. We are informed that Abraham, Chayes, and Chayes have independently found results closely related to (1.18) and (1.19) which describe the decay of connected correlations of an Ising model in three or more dimensions near a surface, at low temperatures.

4) We are indebted to Jean Bricmont for having explained to us techniques useful for the proof of (1.18) and (1.19).

(2) Behaviour of Spin-Spin Correlations Near a Surface in the Kosterlitz-Thouless Phase. We consider the spin-spin correlation

$$
\left\langle S(x) S(y)=\left\langle e^{i(\phi(x)-\phi(y))}\right\rangle, \quad x, y \text { near } \Sigma,\right.
$$

in the Kosterlitz-Thouless phase, II, of the three-dimensional $X Y$ model $(n=2)$ on $\mathbb{L}$, i.e. for $K \ll K_{c}^{3}, J>J_{K T}$. For this model we can prove

(c) If $K \ll K_{c}^{3}$, then

$$
0 \leqq\langle S(x) S(y)\rangle \leqq \text { const }|x-y|^{-\alpha},
$$

for some $\alpha=\alpha(J, K)>0$, with $\alpha(J, K)=0\left(\frac{1}{J}\right)$, as $J \rightarrow \infty$.

(d) If $J \gg J_{K T}$, then

$$
\langle S(x) S(y)\rangle \geqq \text { const }|x-y|^{-\alpha^{\prime}},
$$

for some $\alpha^{\prime}=\alpha^{\prime}(J, K)>0$, with $\alpha^{\prime}(J, K)=0\left(\frac{1}{J}\right)$, as $J \rightarrow \infty$.

Remark. Result (c) is a rather straightforward generalization of the McBryanSpencer bound [15], and the proof is sketched in Sect. 2.3. Result (d) follows from the bounds in [16] by using Ginibre inequalities [17]; see Sect. 3.

(3) Spin-Spin Correlations Near an Ordered Surface. Next, we propose to study the behaviour of $\langle S(x) ; S(y)\rangle$, $x$ and $y$ near $\Sigma$, for values of $K$ deep inside phase III, i.e. $K>K_{c}^{3}$. In the Ising model, for $J \gg J_{c}^{2}$ (the critical coupling of the two-dimensional Ising model) and either $K$ very small, or $K$ very large, $\langle S(x) ; S(y)\rangle$ decays exponentially in $|x-y|, x, y$ in $\Sigma$, as follows from standard low-temperature expansions. Power law corrections have been studied by D. Abraham, J. T. Chayes, and L. Chayes, with results similar to those summarized in (1.18) and (1.19). When $K$ passes through the transition point of the bulk the behaviour of $\langle S(x) ; S(y)\rangle$ is not known precisely, but the power corrections for $J \gg J_{c}^{2}, K=0$, are different from those for $J \gg J_{c}^{2}, K \neq 0$; see e.g. [18].

For the classical $X Y$ model we prove the existence of spontaneous magnetization when $K$ is large enough (phase III); see Sect. 3: If a symmetry breaking magnetic field, $h$, in the 1-direction is turned off then a spontaneous magnetization $\left\langle S^{1}(x)\right\rangle>0$ remains. By the Goldstone-Mermin-Wagner theorem [1,2], $\left\langle S^{2}(x) S^{2}(y)\right\rangle$ must then be expected to have slow decay in $|x-y|$, and this is indeed the case.

For example, one can prove that $\left\langle S^{2}(x) S^{2}(y)\right\rangle, x \in \sum, y \in \mathbb{L}$, decays slowly in $|x-y|$, in an average sense with respect to $y$. This follows in a simple way from the positivity of the spontaneous magnetization, a "Ward identity" for $\left\langle S^{1}(x)\right\rangle$ and correlation inequalities; see [19] for similar results. More precisely, let $\Omega$ be some 
half sphere in $\mathbb{L}$ centered at $x$, and let $\partial \Omega$ be the set of nearest neighbours $y, y^{\prime}$, with $y \in \Omega, y^{\prime} \notin \Omega$. By a simple integration by parts one finds

$$
\begin{aligned}
& 0<\left\langle S^{1}(x)\right\rangle=J \sum_{\left\langle y, y^{\prime}\right\rangle \in \partial \Omega \cap \Sigma}\left\langle S^{2}(x)\left(S^{2}(y) S^{1}\left(y^{\prime}\right)-S^{1}(y) S^{2}\left(y^{\prime}\right)\right)\right\rangle \\
& +K \sum_{\left\langle y, y^{\prime}\right\rangle \in \partial \Omega \backslash \Sigma}\left\langle S^{2}(x)\left(S^{2}(y) S^{1}\left(y^{\prime}\right)-S^{1}(y) S^{2}\left(y^{\prime}\right)\right)\right\rangle \\
& \leqq\left\langle S^{1}\right\rangle J \sum_{\left\langle y, y^{\prime}\right\rangle \in \partial \Omega \Omega \cap \Sigma}\left\langle S^{2}(x) S^{2}(y)\right\rangle \\
& +\left\langle S^{1}\right\rangle K \sum_{\left\langle y, y^{\prime}\right\rangle \in \partial \Omega \backslash \Sigma}\left\langle S^{2}(x) S^{2}(y)\right\rangle,
\end{aligned}
$$

by omitting negative terms and bounding positive terms by correlation inequalities, [20]. Here

$$
\left\langle S^{1}\right\rangle=\sup _{y}\left\langle S^{1}(y)\right\rangle .
$$

Now, since the proof of spontaneous magnetization (a straightforward adaptation of [6]) shows that, for large $K$, Gaussian spin wave theory is correct, up to small corrections, it is reasonable to conjecture that

$$
\left\langle S^{2}(x) S^{2}(y)\right\rangle_{|x-\tilde{y}| \rightarrow \infty} \operatorname{const} K^{-1} G^{N}(x, y),
$$

for large $K$, where $G^{N}(x, y)$ is the Green's function of the finite difference Laplacian, $\Delta^{N}$, with Neumann boundary conditions at $\Sigma$, but we have no rigorous proof of this.

Whatever the exact behaviour of $\left\langle S^{2}(x) S^{2}(y)\right\rangle$, the behaviour of $\left\langle S^{1}(x) ; S^{1}(y)\right\rangle$ is constrained by the inequality

$$
\left\langle S^{1}(x) ; S^{1}(y)\right\rangle \geqq \text { const }\left\langle S^{2}(x) S^{2}(y)\right\rangle^{2},
$$

due to Dunlop and Newman [21].

\section{Absence of Ordering on the Surface $\Sigma$}

\subsection{Effective Hamiltonian}

We compute the effective Hamiltonian when $K$ is small. We choose a specific system, the $X Y$ model with Hamiltonian $(1.1)(n=2)$. We use the parametrization of the spins by angles and normalize the angles so that (1.1) becomes

$$
-\sum_{x, y} K(x, y) \cos 2 \pi(\phi(x)-\phi(y))
$$

and $\phi(x) \in[0,1)$, $\bmod 1$. Any pair $\{x, y\}$ of nearest neighbours is a bond $b=b(x, y)$. The coupling constants are therefore $K(x, y) \equiv K(b)=K$ if $b \nsubseteq \Sigma, K(b)=J$ if $b \subset \Sigma$ and $K(x, y)=0$ otherwise. Let $\Omega_{L}$ be the finite box $\subset \mathbb{L}$

$$
\Omega_{L}=\left\{x \in \mathbb{L}:\left|x^{i}\right| \leqq L, i=1,2, \ldots, d-1, x^{d} \leqq L\right\} .
$$

We fix a configuration of spins outside $\Omega_{L}$, i.e. on $\Omega_{L}^{c}=\mathbb{L} \backslash \Omega_{L}$, and denote it by $\varphi\left(\Omega_{L}^{c}\right)$. We can write the Boltzmann factor in $\Omega_{L}$ for (2.1) with the boundary condition $\varphi\left(\Omega_{L}^{c}\right)$ as

$$
\prod_{b \cap \Omega_{L} \neq \emptyset} I(b)=\prod_{\substack{b \cap \Omega_{L} \neq \emptyset \\ b \subset \Sigma}} I(b) \prod_{\substack{b \cap \Omega_{L} \neq \emptyset \\ b \notin \Sigma}} I(b),
$$


where

$$
I(b)=I(b(x, y))=\exp (K(x, y) \cos 2 \pi(\varphi(x)-\varphi(y))) \equiv 1+U(b) .
$$

Since $K$ is small we write $I(b)=1+U(b)$ for any $b$ such that $K(b)=K$. We apply the standard polymer formalism [22] to exponentiate the second product on the righthand side of (2.3), which is the product over the $b$ 's such that $K(b)=K$ and $b \cap \Omega_{L} \neq \emptyset$. Let $X \subset \mathbb{L}$, and let $\gamma$ be a graph, whose vertices are in $V(\gamma) \subset \mathbb{L}$ and with bonds in $B(\gamma)$. We say that $X \subset \mathbb{L},|X| \geqq 2$, is connected if the graph $\gamma$, with vertices $V(\gamma)=X$ and bonds $B(\gamma)=\{$ bonds $b \subset \gamma\} \subseteq B(X)$, (the set of all bonds on $X$ ), is connected. $(|X|$ is the cardinality of $X$.) Let $X \subset \mathbb{L},|X| \geqq 2$, be finite and connected. We introduce

$$
U_{L}(X)=\sum_{\gamma} \prod_{b \in B(\gamma)} U(b)
$$

where the sum extends over all connected graphs $\gamma$ with $V(\gamma)=X$ and whose bonds are bonds, $b$, in $B(X)$ such that $K(b)=K$ and $b \cap \Omega_{L} \neq \emptyset$. In all other cases $U_{L}(X)=0$. Using the $U$-function we can write

$$
\prod_{\substack{b \cap \Omega_{L} \neq \emptyset \\ b \notin \Sigma}} I(b)=\prod_{\substack{b \cap \Omega_{L} \neq \emptyset \\ K(b)=K}}(1+U(b))=1+\sum_{n \geqq 1} \frac{1}{n !} \sum_{X_{1}, \ldots, X_{n}} \prod_{i=1}^{n} U_{L}\left(X_{i}\right),
$$

where the sum extends over all families of $n$ disjoint subsets. Let $Q_{L}(X)$ be the result of the integration of $U_{L}(X)$ with respect to the angles $\varphi(y)$, with $y \in X \cap\left(\Omega_{L} \backslash \Sigma\right)$. Let $\psi\left(X_{1}, \ldots, X_{n}\right)=1$ if all the subsets $X_{i}$ are disjoint, and $\psi\left(X_{1}, \ldots, X_{n}\right)=0$, otherwise. After integration of the spins $\varphi(y)$, with $y \in \Omega_{L} \backslash \Sigma$, we get for (2.6)

$$
1+\sum_{n \geqq 1} \frac{1}{n !} \sum_{X_{1}, \ldots, X_{n}} \prod_{i=1}^{n} Q_{L}\left(X_{i}\right) \psi\left(X_{1}, \ldots, X_{n}\right)
$$

Let

$$
C(b)=\left\|e^{K(b) \cos 2 \pi \varphi}-1\right\|_{\infty} .
$$

Notice that $C(b)$ bends to zero if $K(b)$ tends to zero. We have

$$
\left|Q_{L}(X)\right| \leqq 2^{d|X|} C(b)^{d|X|} \equiv \gamma_{d}(K)^{|X|}
$$

and

$$
\sum_{\substack{X \ni 0 \\ X \subset \mathbb{Z}^{d}}}\left|Q_{L}(X)\right| e^{|X|} \leqq \sum_{n \geqq 1} e^{n}\left(\gamma_{d}(K)\right)^{n} \alpha_{d}^{n}=\frac{e \cdot \gamma_{d} \cdot \alpha_{d}}{1-e \cdot \gamma_{d} \cdot \alpha_{d}}
$$

where $\alpha_{d}^{n}$ is an upper bound for the number of subsets $X \subset \mathbb{Z}^{d}$ with $X \ni 0$ and $|X|=n$. Provided that (2.10) is smaller than 1, we can write (2.7), using the Mayer expansion, as

$$
\exp \left(\sum_{n \geqq 1} \frac{1}{n !} \sum_{X_{1}, \ldots, X_{n}} \prod_{i=1}^{n} Q_{L}\left(X_{i}\right) \psi_{c}\left(X_{1}, \ldots, X_{n}\right)\right)
$$


where the factor $\psi_{c}\left(X_{1}, \ldots, X_{n}\right)$ is a well-known combinatorial coefficient (see e.g. [22]). We can now read off the effective interaction. Let $Y \subset \Sigma \cap \Omega_{L}$, and define

$$
\phi_{L}(Y)=\sum_{n \geqq 1} \frac{1}{n !} \sum_{X_{1}, \ldots, X_{n}} \prod_{i=1}^{n} Q_{L}\left(X_{i}\right) \psi_{c}\left(X_{1}, \ldots, X_{n}\right),
$$
where the second sum extends over all $X_{1}, \ldots, X_{n}$, such that $\left(\bigcup_{i=1}^{n} X_{i}\right) \cap \Sigma=Y$. If
(2.10) is smaller than one,

$$
\sum_{Y \ni 0}\left|\phi_{L}(Y)\right| \leqq C
$$

with $C$ independent of $L$. We can write (2.11) as

$$
\exp \left(\sum_{Y \subset \Sigma_{L}} \phi_{L}(Y)+R_{L}\right)
$$

where $\Sigma_{L}=\Omega_{L} \cap \Sigma$ and $R_{L}$ is independent of $\varphi(x), x \in \Sigma_{L}$. The expectation value of any local observable on $\Sigma_{L}$ is given by the Gibbs formula for a spin model defined on $\Sigma_{L}$ with Hamiltonian

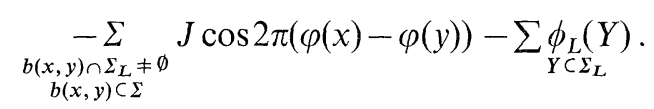

Remark. $\phi_{L}(Y)$ depends on the boundary condition $\varphi\left(\Omega_{L}^{c}\right)$. We can always decompose $\phi_{L}(Y)$ as

$$
\phi_{L}(Y)=\phi_{1, L}(Y)+\phi_{2, L}(Y),
$$

where $\phi_{1, L}(Y)$ does not depend on $\varphi\left(\Omega_{L}^{c}\right)$ by definition. Thus $\phi_{1, L}(Y)$ is invariant under a rotation of all $\varphi(x), x \in Y$ by the same angle. Let $\delta, 0<\delta<1$, and $K$ so small that

$$
\sum_{\substack{X \ni 0 \\ X \subset \mathbb{Z}^{d}}}\left|Q_{L}(X)\right| \delta^{-|X|} e^{|X|}<1
$$

Then

$$
\sum_{\substack{X \subset \Sigma_{L} \\ X \ni x}}\left\|\phi_{2, L}(X)\right\|_{\infty} \leqq \operatorname{const} \delta^{\mathrm{dist}\left(x, \Sigma \backslash \Sigma_{L}\right)}
$$

with a constant independent of $L$.

\subsection{Absence of Symmetry Breakdown}

We take again $d=3$ and we choose $K$ small enough to ensure the validity of (2.17) and (2.27) below. We want to prove that any Gibbs state obtained by taking the thermodynamic limit of a model with Hamiltonian (2.15) is necessarily invariant under the internal symmetry group, which is the circle group $G$ in our specific example. We use the method of [3]. In this method we must find a uniform upper bound on the relative entropy of a perturbed equilibrium state with respect to the unperturbed equilibrium state. The unperturbed equilibrium state can be constructed as the thermodynamic limit of finite volume Gibbs states. Therefore we work with finite volume Gibbs states and try to find a bound which is also 
uniform in the volume. Let $\mu_{L}$ be a Gibbs state on $\Sigma_{L}$ with Hamiltonian (2.15) and some boundary condition. The perturbed equilibrium state $\mu_{L}^{\prime}$ is obtained from $\mu_{L}$ by the following transformation. We choose an arbitrary positive number $l$, and choose $L \gg 2 l$. Let $g \in G$ and consider the transformation $R(g): \varphi(x) \rightarrow \varphi(x)+g(x)$ given by

$$
\begin{array}{ll}
g(x)=g & \text { if } \max \left(\left|x^{1}\right|,\left|x^{2}\right|\right) \leqq l, \\
g(x)=g \cdot \frac{l-k}{k} & \text { if } \max \left(\left|x^{1}\right|,\left|x^{2}\right|\right)=l+k, \quad 0<k \leqq l, \\
g(x)=0, & \text { otherwise. }
\end{array}
$$

This is a local transformation and inside

$$
\Sigma_{l}=\left\{x \in \Sigma:\left|x^{1}\right| \leqq l,\left|x^{2}\right| \leqq l\right\}
$$

it is a rotation by $g$. Let $\mu_{L}^{\prime}=R(g) \mu_{L}$ [for any observable $\left.\mu_{L}^{\prime}(f)=\mu_{L}(f \circ R(g))\right]$. If there is an upper bound, $C$, uniform in $L, l$ and the boundary condition,

$$
0 \leqq S\left(\mu_{L}^{\prime} \mid \mu_{L}\right)=-\int d \mu_{L} \log \frac{d \mu_{L}^{\prime}}{d \mu_{L}} \leqq C<\infty,
$$

then all equilibrium states for $L \rightarrow \infty$ are rotation invariant; (see [3]). Technically it is simpler to estimate

where $\mu_{L}^{\prime \prime}=R(g)^{-1} \mu_{L}$.

$$
S\left(\mu_{L}^{\prime} \mid \mu_{L}\right)+S\left(\mu_{L}^{\prime \prime} \mid \mu_{L}\right)
$$

Let $X \subset \Sigma_{L}, X=\left\{x_{1}, \ldots, x_{n}\right\}$. Let

$$
\varphi(X)=\left\{\varphi\left(x_{1}\right), \ldots, \varphi\left(x_{n}\right)\right\} \quad \text { and } \quad g(X)=\left\{g\left(x_{1}\right), \ldots, g\left(x_{n}\right)\right\} .
$$

We write $\varphi(X)+g(X)=\left\{\varphi\left(x_{1}\right)+g\left(x_{1}\right), \ldots, \varphi\left(x_{n}\right)+g\left(x_{n}\right)\right\}$. Since $L$ is fixed we write $\phi(X)$ instead of $\phi_{L}(X)$. We also introduce $\phi^{\prime}(X)=\phi(\varphi(X)-g(X))$ and $\phi^{\prime \prime}(X)$ $=\phi(\varphi(X)+g(X))$. It is easy to compute $(2.21)$ :

$$
\begin{aligned}
S\left(\mu_{L}^{\prime} \mid \mu_{L}\right)+S\left(\mu_{L}^{\prime \prime} \mid \mu_{L}\right)= & \int d \mu_{L} \sum_{\substack{b=b(x, y) \\
b \cap \Sigma_{2 l} \neq \emptyset\\
}} J[2 \cos 2 \pi(\varphi(x)-\varphi(y))-\cos 2 \pi(\varphi(x)-\varphi(y) \\
& -g(x)+g(y))-\cos 2 \pi(\varphi(x)-\varphi(y)+g(x)-g(y))] \\
+ & \int d \mu_{L} \sum_{\substack{x \subset \Sigma_{L} \\
X \cap \Sigma_{2 l} \neq \emptyset}}\left(2 \phi(X)-\phi^{\prime}(X)-\phi^{\prime \prime}(X)\right) .
\end{aligned}
$$

Let us consider the most difficult terms in (2.22). We know that $\phi=\phi_{1}+\phi_{2}$. Using (2.18) and choosing $L$ large enough, the contribution of the terms $\phi_{2}$ to (2.22) can be made arbitrarily small. Therefore we have to worry only about

$$
\sum_{\substack{X \subset \Sigma_{L} \\ X \cap \Sigma_{2 l} \neq \emptyset}} 2 \phi_{1}(X)-\phi_{1}^{\prime}(X)-\phi_{1}^{\prime \prime}(X) .
$$

$\phi_{1}(X)$ is invariant by rotation. Therefore

$$
\phi_{1}^{\prime}(X)=\phi_{1}\left(\varphi\left(x_{1}\right), \varphi\left(x_{2}\right)+g\left(x_{1}\right)-g\left(x_{2}\right), \ldots, \varphi\left(x_{n}\right)+g\left(x_{1}\right)-g\left(x_{n}\right)\right),
$$

and a similar expression holds for $\phi_{1}^{\prime \prime}(X)$. The interaction is smooth; we use only the fact that it is $C^{2}$ in order to make a Taylor expansion with remainder. The first 
order terms in (2.23) cancel exactly. Therefore we get the upper bound

$$
\begin{aligned}
\left|2 \phi_{1}(X)-\phi_{1}^{\prime}(X)-\phi_{1}^{\prime \prime}(X)\right| \leqq & \sum_{j=2}^{n} \sum_{k=2}^{n}\left\|\frac{\partial^{2} \phi_{1}}{\partial \varphi\left(x_{j}\right) \partial \varphi\left(x_{k}\right)}\right\| \|_{\infty}\left|g\left(x_{1}\right)-g\left(x_{j}\right)\right| \\
& \times\left|g\left(x_{1}\right)-g\left(x_{k}\right)\right| .
\end{aligned}
$$

From the definition of $R(g)$ we have

Therefore let

$$
\left|g\left(x_{i}\right)-g\left(x_{j}\right)\right| \leqq \frac{1}{l} \operatorname{dist}\left(x_{i}, x_{j}\right) .
$$

$$
\begin{aligned}
\left\|\phi_{1}(X)\right\| \|= & \sup _{x_{i} \in X} \sum_{j \neq i} \sum_{k \neq i}\left\|\frac{\partial^{2} \phi_{1}}{\partial \varphi\left(x_{j}\right) \partial \varphi\left(x_{k}\right)}\right\|_{\infty} \\
& \operatorname{dist}\left(x_{i}, x_{j}\right) \operatorname{dist}\left(x_{i}, x_{k}\right) .
\end{aligned}
$$

If $K$ is small enough, we have

$$
\sum_{\substack{X \ni 0 \\ X \subset \Sigma}}\left\|\phi_{1}(X)\right\| \| \text { const }<\infty
$$

and the constant is independent of $L$. We have immediately a uniform bound for (2.23)

$$
\sum_{\substack{X \subset \Sigma_{L} \\ X \cap \Sigma_{2 l} \neq \emptyset}}\left\|2 \phi_{1}(X)-\phi_{1}^{\prime}(X)-\phi_{1}^{\prime \prime}(X)\right\|_{\infty} \leqq \sum_{x \in \Sigma_{2 l}} \sum_{X \ni x}\left\|\phi_{1}(X)\right\| \cdot\left(\frac{1}{l}\right)^{2} \leqq \text { const, }
$$

[see (2.24) and (2.25)]. This, combined with standard arguments [3], yields (2.20).

\subsection{McBryan-Spencer Bound}

In this subsection we show that if $0 \leqq K \ll K_{c}^{3}$, and for $x \in \Sigma$,

$$
0 \leqq\langle S(0) S(x)\rangle=\left\langle e^{2 \pi i(\varphi(0)-\varphi(x))}\right\rangle \leqq \text { const }|x|^{-\alpha},
$$

for some positive $\alpha=\alpha(J, K)=0\left(\frac{1}{J}\right)$. See Sect. 1, (1.21). The lower bound in (2.29) follows from Ginibre's inequalities [17]. To prove the upper bound, we use the method of complex translations of the angular variables, $\varphi(j)$, introduced in [15] and the effective Hamiltonian (2.15). Our arguments are very similar to those in Subsects. 2.1 and 2.2, so we only sketch them.

Let $j \in \mathbb{L}$, and set $|j|=\max \left(\left|j^{1}\right|,\left|j^{2}\right|\right)$. Instead of the real translations, $\varphi(j) \rightarrow \varphi(j)$ $+g(j)$, introduced in (2.19), we perform complex translations,

$$
\varphi(j) \rightarrow \varphi(j)+i a_{x}(j)
$$

where $a_{x} \equiv a$ is defined by

$$
\begin{array}{ll}
a(j)=0 & \text { if }|j|>|x|, \\
a(j)=C \cdot \sum_{k=0}^{n} \frac{1}{|x|-k+1} & \text { if }|j|=|x|-n .
\end{array}
$$


From the observable, $\exp 2 \pi i(\varphi(0)-\varphi(x))$ we obtain, after the change of variables (2.30), a small factor

where

$$
\exp \left(-\frac{\lambda}{J} L(|x|)\right)
$$

$$
L(|x|)=\sum_{k=0}^{|x|-1} \frac{1}{k+1} \sim \log |x|
$$

and we have chosen $C=\frac{\lambda}{2 \pi J}$ in the definition of $a(j)$. Next we must prove an upper bound on

$$
G(0, x) \equiv\langle\exp 2 \pi i(\varphi(0)-\varphi(x)) \exp (H(\varphi)-H(\varphi+i a))\rangle^{+},
$$

where $H$ is the Hamiltonian of the model. We re-write (2.33) as follows:

where

$$
\begin{aligned}
G(0, x)= & \lim \int \prod_{L \rightarrow \infty} d \varphi(j) \exp 2 \pi i(\varphi(0)-\varphi(x)) Z_{\Omega_{L}}^{-1} \prod_{b \cap \Sigma_{L} \neq \emptyset} I_{a}(b) \\
& \times \int \prod_{j \in \Omega_{L} \backslash \Sigma_{L}} d \varphi(j) \prod_{\substack{b \cap \Omega_{L} \neq \emptyset \\
b \subset \Sigma_{L}}} I_{a}(b),
\end{aligned}
$$

$$
I_{a}(b)=\exp \{K(j, l) \cos 2 \pi[\varphi(j)-\varphi(l)+i(a(j)-a(l))]\},
$$

with $K(j, l)=J$ if $j$ and $l$ belong to $\Sigma$, and $=K$, otherwise, and where $a(j)$ is defined by $(2.31)$.

We now note that, for $j$ and $l$ nearest neighbours,

$$
|a(j)-a(l)| \leqq \frac{\lambda}{J} \frac{1}{\min (|j|,|l|)+1} \leqq \frac{\lambda}{J} .
$$

This bound is uniform in $x$ and in $j, l$. It follows that

$$
C_{a}(b) \equiv\left\|U_{a}(b)\right\|_{\infty} \leqq \exp K \cosh 2 \pi\left(\frac{\lambda}{J}\right)-1 \rightarrow 0,
$$

as $K \rightarrow 0$. Furthermore, if $X \cap \Sigma=\emptyset$

where

$$
Q_{L, a}(X)=\int \prod_{j \in X} d \varphi(j) U_{L, a}(X)=Q_{L}(X)
$$

and

$$
U_{L, a}(X)=\sum_{\substack{\gamma: V(\gamma)=X \\ \gamma \text { connected }}} \prod_{b \in \gamma} U_{a}(b)
$$

$$
U_{a}(b)=I_{a}(b)-1
$$

Equation (2.37) follows from the periodicity of the cosine by shifting the integration contour for $\varphi(j)$ by $-i a(j)$. It follows from (2.36) and (2.37) that the expansion of Subsect. 2.1 still converges if $I(b)$ is replaced by $I_{a}(b)$, for every $b$, etc., provided $|K|<K\left(\frac{\lambda}{J}\right)$, for some positive number $K\left(\frac{\lambda}{J}\right)$ which only depends on $\lambda$ 
and $J$, but not on our choice of $x$. Let $Y \subset \Sigma \cap \Omega_{L}$, and define

$$
\phi_{L, a}(Y)=\sum_{n \geqq 1} \frac{1}{n !} \sum_{X_{1}, \ldots, X_{n}} \prod_{i=1}^{n} Q_{L, a}\left(X_{i}\right) \psi_{c}\left(X_{1}, \ldots, X_{n}\right)
$$
where the second sum extends over all $X_{1}, \ldots, X_{n}$ such that $\left(\bigcup_{i=1}^{n} X_{i}\right) \cap \Sigma=Y$. We
also define

$$
H_{a}^{\mathrm{eff}}(\varphi)=-\sum_{\substack{b(j, l) \cap \Sigma_{L} \neq \emptyset \\ b(j, l) \subset \Sigma}} J \cos 2 \pi[\varphi(j)-\varphi(l)+i(a(j)-a(l))]-\sum_{Y \subset \Sigma_{L}} \phi_{L, a}(Y) .
$$

Then

$$
\begin{aligned}
& G(0, x) \lim _{L \rightarrow \infty} Z_{L, a}^{-1} \int \prod_{j \in \Sigma_{L}} d \varphi(j) \exp 2 \pi i(\varphi(0)-\varphi(x)) \exp \left[-H_{L, a}^{\mathrm{eff}}(\varphi)\right] \\
& \quad=\lim _{L \rightarrow \infty}\left\langle e^{2 \pi i(\varphi(0)-\varphi(x))} \exp \left[H_{L, 0}^{\mathrm{eff}}(\varphi)-H_{L, a}^{\mathrm{eff}}(\varphi)\right]\right\rangle_{L}^{+} .
\end{aligned}
$$

We Taylor-expand $H_{L, 0}^{\mathrm{eff}}(\varphi)-H_{L, a}^{\mathrm{eff}}(\varphi)$ to second order in $a$, noticing that the modulus of the exponential of the first order terms is equal to one. Let $X \subset \Sigma$, and let $y(X)$ denote the site in $X$ closest to the origin, 0 . (If there are several such points, we choose the smallest one in lexicographic order.) Let $y(X)=i \in X, j \in X$. Then

$$
|a(i)-a(j)| \leqq \frac{\lambda}{J} \sum_{k=|i|}^{|j|-1} \frac{1}{k+1} \leqq \frac{\lambda / J}{|i|+1}|j-i|
$$

In estimating the modulus of the second order remainder of $H_{L, 0}^{\mathrm{eff}}(\varphi)-H_{L, a}^{\mathrm{eff}}(\varphi)$, the nearest-neighbor terms yield a contribution of order

$$
O\left(\frac{\lambda^{2}}{J}\right) \log |x| \text {. }
$$

The terms depending on $\phi_{2}(X)$ tend to 0 exponentially, as $L \rightarrow \infty$, for every $x$, and hence can be ignored. Finally, we must estimate a term analogous to (2.23). When summing over $X$ we first sum over all $X$ with $y(X) \equiv y$ fixed. This yields a contribution bounded by

$$
\text { const } K \frac{\lambda^{2}}{J^{2}}(|x|-|y|+1)^{-2},
$$

see (2.24), (2.25), (2.35), and (2.40). The sum of all these contributions over all $y$, with $|y| \leqq|x|$, is therefore bounded by $O\left(K \frac{\lambda^{2}}{J^{2}}\right) \log |x|$. This, together with (2.41) and (2.32), completes the proof of the upper bound in (2.29) by choosing $\lambda$ small enough.

\section{Existence of Ordering on the Surface}

For the $X Y$ model, with $J=K$, we can prove that, for large $K$, there is a spontaneous magnetization on $\Sigma$. The method is that of [6] which is also described in $[25$, Sect. 8.5]. This proof, even for the Villain model, is relatively long. Since it is 
essentially the same in our case as in [25], except for minor details, we do not reproduce it. Let us however describe the main ideas. It is sufficient to prove that there exists a positive constant $C>0$, such that for large $K$

$$
\langle\exp 2 \pi i(\varphi(0)-\varphi(x))\rangle^{+} \geqq C>0
$$

where $x \in \Sigma$ is arbitrary. The state $\langle\cdot\rangle^{+}$is clustering, and we can conclude that there is a spontaneous magnetization. In order to prove (3.1) we use a duality transformation to express the model as a model of spin waves and vortices. In the Villain model there is no direct interaction between spin waves and vortices, and this simplifies the analysis somewhat. ${ }^{1}$ If the vortices were absent the result (3.1) would be true for any $K>0$ : In our case the spin wave part is a Gaussian measure whose Boltzmann factor is

$$
\exp \left(\frac{K}{2} \sum_{x} \theta(x)(\Delta \theta)(x)\right) .
$$

In (3.2) $\theta(x) \in \mathbb{R}$ and $\Delta$ is the lattice Laplacian on $\mathbb{L}$ with Neumann boundary condition on $\Sigma$. The main part of the proof is to show that the vortices do not destroy the effect of the spin waves for large values of $K$. This is a kind of Peierls argument, more complicated than the usual Peierls argument in the Ising model, which is used to show that the defects in the system do not destroy the ordering present in the ground states. The only differences between the situation analyzed in [6] and the present one is that, in the situation studied here, vortices can terminate on the boundary, $\Sigma$, of $\mathbb{L}$ and that the finite difference operators appearing in the estimates now have to obey specific boundary conditions on $\Sigma$. But these differences are minor and do not warrant presenting a detailed proof.

We show that for $K>K^{*}$ and any $J$ there is always an ordering on the surface. By definition of $K^{*},\langle\cos \varphi(0)\rangle^{+}(K, K)>0$ if $K>K^{*}$. Therefore it is sufficient to show that $\langle\cos \varphi(0)\rangle^{+}(J=0, K)>0$, for $K>K^{*}$. In this expectation the spin $S(0)$ is only coupled directly to $S(x)$, with $x=(0,0,1)$. It is easy to compute the conditional expectation value of $\cos \varphi(0)$, given the value of $\varphi(x)$. We find

$$
\cos \varphi(x) \frac{I_{1}(K)}{I_{0}(K)}
$$

where $I_{n}(K)$ is the $n^{\text {th }}$ modified Bessel function. By Ginibre inequalities [17]

$$
\langle\cos \varphi(x)\rangle^{+}(J=0, K) \geqq\langle\cos \varphi(0)\rangle^{+}(K, K),
$$

and therefore

$$
\langle\cos \varphi(0)\rangle^{+}(J, K) \geqq\langle\cos \varphi(0)\rangle^{+}(J=0, K) \geqq \frac{I_{1}(K)}{I_{0}(K)}\langle\cos \varphi(0)\rangle^{+}(K, K) .
$$

\footnotetext{
${ }^{1}$ The usual $X Y$ model can be analyzed too, but the proof is somewhat more complicated; see Sect. 6 of [6]
} 


\section{Phase Diagram for the Ferromagnetic $X Y$ Model}

\subsection{Boundary Susceptibility $\chi_{\Sigma}$}

We use an argument given in [9] in order to analyze $\chi_{\Sigma}$. We leave out some minor technical points. Details can be found in $[8,9]$. In order to simplify our notation, we write $\langle 0, x\rangle$ or $\langle x y z\rangle$ instead of $\langle\cos \varphi(0) \cos \varphi(x)\rangle^{+}$or

$$
\langle\cos \varphi(x) \cos \varphi(y) \cos \varphi(z)\rangle^{+} .
$$

We compute

$$
\frac{d}{d K(x, y)}\langle z w\rangle \leqq\langle z w x y\rangle-\langle z w\rangle\langle x y\rangle,
$$

where we have used the inequality [20],

$\langle\cos \varphi(z) \cos \varphi(w) \sin \varphi(x) \sin \varphi(y)\rangle^{+} \leqq\langle\cos \varphi(z) \cos \varphi(w)\rangle^{+}\langle\sin \varphi(x) \sin \varphi(y)\rangle^{+}$.

We have

$$
\begin{aligned}
& \langle z w x y\rangle-\langle z w\rangle\langle x y\rangle=\langle z w x y\rangle-\langle z w\rangle\langle x y\rangle-\langle z x\rangle\langle w y\rangle-\langle z y\rangle\langle w x\rangle \\
& +\langle z x\rangle\langle w y\rangle+\langle z y\rangle\langle w x\rangle \leqq\langle z x\rangle\langle w y\rangle+\langle z y\rangle\langle w x\rangle
\end{aligned}
$$

if $\langle x\rangle=0$, [23]. We apply (4.3) for $K<K^{*}$.

$$
\begin{aligned}
\frac{d}{d J} \chi_{\Sigma} & =\frac{d}{d J} \sum_{z \in \Sigma}\langle 0 z\rangle \leqq \sum_{z \in \Sigma} \sum_{\substack{\{x, y\}) \subset \Sigma \\
\operatorname{dist}(x, y)=1}}(\langle 0 y\rangle\langle x z\rangle+\langle 0 x\rangle\langle y z\rangle) \\
& =2 \sum_{\{x, y\}}\langle 0 y\rangle \chi_{\Sigma}=4\left(\chi_{\Sigma}\right)^{2} .
\end{aligned}
$$

By integrating (4.4) we get

$$
\chi_{\Sigma}^{-1}\left(J_{1}, K\right)-\chi_{\Sigma}^{-1}\left(J_{2}, K\right) \leqq 4\left(J_{2}-J_{1}\right), \quad J_{2} \geqq J_{1} .
$$

The function $J_{K T}(K)$ is defined by

$$
J_{K T}(K)=\inf \left\{J: \chi_{\Sigma}^{-1}(J, K)=0\right\} .
$$

If $K \rightarrow J_{K T}(K)$ is the graph of a function, then by continuity of $J \rightarrow \chi_{\Sigma}^{-1}(J, K)$ we must have $\chi_{\Sigma}^{-1}\left(J_{K T}(K), K\right)=0$. Thus in this case the boundary $\Gamma_{2}$ is critical. Let us assume that we cross $\Gamma_{2}$ at $(\hat{J}, \hat{K})$ along the straight line

$$
\left\{(J, K): J=\beta J_{0}, K=\beta K_{0}, \beta \geqq 0\right\} .
$$

Let $\hat{\beta}$ be given by $\hat{J}=\hat{\beta} J_{0}, \hat{K}=\hat{\beta} K_{0}$. From (4.5) we get

$$
\begin{aligned}
\chi_{\Sigma}^{-1}\left(\beta J_{0}, \beta K_{0}\right) & =\chi_{\Sigma}^{-1}\left(\beta J_{0}, \beta K_{0}\right)-\chi_{\Sigma}^{-1}\left(J_{K T}\left(\beta K_{0}\right), \beta K_{0}\right) \leqq 4\left(J_{K T}\left(\beta K_{0}\right)-\beta J_{0}\right) \\
& =4\left(J_{K T}\left(\beta K_{0}\right)-\hat{\beta} J_{0}+(\hat{\beta}-\beta) J_{0}\right) \\
& =4\left(J_{K T}\left(\beta K_{0}\right)-J_{K T}\left(\hat{\beta} K_{0}\right)+(\hat{\beta}-\beta) J_{0}\right) .
\end{aligned}
$$

If $K \rightarrow J_{K T}(K)$ is Lipshitz-continuous at $\hat{K}$, we see from (4.6) that $\chi_{\Sigma}$ diverges at least like $(\hat{\beta}-\beta)^{-1}$ when $\beta \uparrow \hat{\beta}$. 
We now analyze the behaviour of $\chi_{\Sigma}$ in the neighborhood of $\Gamma_{1}$ [under the assumption that $\left.\Gamma_{1}=\left\{(J, K): K=K_{c}^{3}, J \leqq J^{*}\right\}\right]$. Since $\Gamma_{1}$ is a vertical line, we cannot conclude from (4.5) that $\chi_{\Sigma}^{-1}(J, K)=0$ on $\Gamma_{1}$. However, if we suppose that, for the special choice $J=K$, one has a critical point at $K_{c}^{3}$, with a divergent suceptibility $\chi_{\Sigma}(K, K)$, with critical exponent $\gamma$, we have, by correlation inequalities, that $\chi_{\Sigma}^{-1}\left(J, K_{c}^{3}\right)=0$, for $K_{c}^{3} \leqq J \leqq J^{*}$. Moreover, using the argument of Sect. 3, we see that the same result is true for $J \leqq K_{c}^{3}$, and the critical exponent $\gamma$ is independent of $J$, at least for $J \leqq K_{c}^{3}$. Therefore we have that $\Gamma_{1}$ is also a critical line, under the above assumption.

\subsection{Boundary Correlation Length $\xi_{\Sigma}$}

We define, for $j \in \mathbb{L}$,

$$
\chi_{j}(J, K)=\sum_{x \in \mathbb{L}}\langle j x\rangle .
$$

The first step of the argument is to show that $\chi_{\Sigma}<\infty$ and $K<K_{c}^{3}$ imply that $\chi_{j}(J, K)<\infty$. Let us consider the case where $j=0$. Exactly as for (4.4)

$$
\frac{d \chi_{0}}{d J} \leqq \sum_{\substack{\{x, y\} \\ \operatorname{dist}(x, y)=1}} \sum_{z \in \mathbb{L}}(\langle 0 y\rangle\langle x z\rangle+\langle 0 x\rangle\langle y z\rangle)=4 \chi_{\Sigma} \chi_{0} .
$$

By integration we find

$$
\chi_{0}\left(J_{2}, K\right) \leqq \chi_{0}\left(J_{1}, K\right) \exp \left\{4 \int_{J_{1}}^{J_{2}} \chi_{\Sigma}\left(J^{\prime}, K\right) d J^{\prime}\right\} .
$$

In (4.9) we choose $J_{1}=K$. Then, by correlation inequalities

$$
\chi_{0}(K, K) \leqq \chi_{X Y}(K)<\infty \text {, }
$$

where $\chi_{X Y}(K)$ is the susceptibility of the 3-dimensional $X Y$ model with coupling constant $K$. [Strictly speaking, $\chi_{X Y}(K)<\infty$ for $K<K_{c}^{3}$ is not known as a mathematical fact. It was assumed in Sect. 1.4.] In the 3-dimensional $X Y$ model with coupling constant $K$ we choose $R$ so large that

$$
\max (J, K) \cdot\left\{\sum_{|y|=R}\langle 0 y\rangle_{\mathbb{Z}^{3}}(K)\right\}<\frac{1}{2}
$$

where $|y|=\max \left\{\left|y^{i}\right|, i=1,2,3\right\}$. Similarly we define, for $x \in \mathbb{L}$,

$$
a(x ; R) \equiv a(x)=\sum_{\substack{y \in \mathbb{I} \\|y-x|=R}}\langle x y\rangle(J, K) .
$$

It is not difficult to prove that the limit of $a(x ; R)$, when $x^{3} \rightarrow \infty$, exists and is equal to $\sum_{|y|=R}\langle 0 y\rangle_{\mathbb{Z}^{3}}(K)$. Indeed, for $J \leqq K, a(x ; R)$ is a monotone increasing function of $x^{3}$. For $J>K$ we add to the model a boundary field on the first layer and let this field go to infinity. We get an upper bound $a^{+}(x ; R)$ for $a(x ; R)$, and $a^{+}(x ; R)$ is monotone decreasing in $x^{3}$. Since there is a unique Gibbs state for the 3-dimensional model when $K<K_{c}^{3}$, we have the result. We can find $L<\infty$ so that, for $x^{3}>L$,

$$
\max (J, K) \cdot a(x, R) \leqq \frac{3}{4} .
$$


For each $x$, with $0 \leqq x^{3} \leqq L$, we choose $R(x)$ so that

$$
\max (J, K) a(x, R(x)) \leqq \frac{3}{4} .
$$

This is possible, since $\chi_{j}(J, K)<\infty$. Let $\bar{R}=\max \left\{R ; R(x), 0<x^{3} \leqq L\right\}$. It is now possible to apply the argument of Simon. Let $\Omega_{R(0)}$ be the box (2.2) and let $x$ be outside $\Omega_{R(0)} \cap \Sigma=\Sigma_{R(0)}$. We have the inequality [20],

$$
\langle 0 x\rangle \leqq \sum_{y \in \Omega_{R(0)}} \sum_{z \notin \Omega_{R(0)}} K(y z)\langle 0 y\rangle\langle x z\rangle \leqq \max (J, K) a(0, R(0))\langle x \hat{z}\rangle,
$$

where $\langle x \hat{z}\rangle$ is the maximum of $\langle x z\rangle$ for $z$ such that $\operatorname{dist}\left(z, \Omega_{R(0)}\right)=1$. If $|x| \geqq n \cdot \bar{R}$, then, by repeating this argument, we get

$$
\langle 0 x\rangle \leqq\left(\frac{3}{4}\right)^{n}, \text { i.e. } \quad \xi_{\Sigma}<\infty .
$$

\subsection{Transverse Correlation Length}

We have the inequality

$$
\begin{aligned}
\langle\cos \varphi(x) \cos \varphi(y)\rangle^{+} & =\left\langle\cos \varphi(x) \cos \varphi(y)\left(\cos ^{2} \varphi(z)+\sin ^{2} \varphi(z)\right)\right\rangle^{+} \\
& \geqq\left\langle\cos \varphi(x) \cos \varphi(y) \cos ^{2} \varphi(z)\right\rangle^{+} \\
& \geqq\langle\cos \varphi(x) \cos \varphi(z)\rangle^{+}\langle\cos \varphi(y) \cos \varphi(z)\rangle^{+} .
\end{aligned}
$$

Let $z=\left(0,0, z^{3}\right)$ and $x_{0}=0, x_{1}=\left(0,0, x_{1}^{3}\right), x_{2}=\left(0,0, x_{2}^{3}\right), \ldots, x_{n}=\left(0,0, x_{n}^{3}\right)$ with

$$
x_{1}^{3}<x_{2}^{3}<\ldots<x_{n}^{3}=z^{3}=L .
$$

Using (4.17) we get

$$
-\frac{1}{L} \ln \langle 0 z\rangle \leqq \sum_{i=1}^{n}-\frac{1}{L} \ln \left\langle x_{i-1} x_{i}\right\rangle
$$

We choose $x_{i}$ so that $x_{i}^{3}-x_{i-1}^{3}=D$, for all $i$.

We replace each $\left\langle x_{i-1} x_{i}\right\rangle$ by $\left\langle x_{i-1} x_{i}\right\rangle_{\mathbb{Z}^{3}}=\langle 0 D\rangle_{\mathbb{Z}^{3}}$, the corresponding expectation value in the bulk equilibrium state (defined with free boundary condition).

To evaluate the error we make, we use the following property: for any $\varepsilon>0$ there exists a subset $\Lambda_{\varepsilon}$ such that, for any $\Lambda \supseteqq \Lambda_{\varepsilon}$, we have

Using this result we get

$$
\left|\langle 0 D\rangle_{\mathbb{Z}^{3}}-\langle 0 D\rangle_{\Lambda}\right| \leqq \varepsilon
$$

$$
\lim _{L \rightarrow \infty}-\frac{1}{L} \ln \langle 0 z\rangle=\left(\xi_{\perp}(J, K)\right)^{-1} \leqq-\frac{1}{D} \ln \langle 0 D\rangle_{\mathbb{Z}^{3}}+0(\varepsilon),
$$

for $\varepsilon$ small enough, depending on $D$. We can choose $\varepsilon$ arbitrarily small and then take the limit $D \rightarrow \infty$. Thus

$$
\xi_{\perp}(J, K) \geqq \xi_{\text {Bulk }}(K) .
$$

[The value of $J$ does not matter since $\left\langle x_{i-1} x_{i}\right\rangle(J=0, K) \leqq\left\langle x_{i-1} x_{i}\right\rangle(K, K)$ $\leqq\left\langle x_{i-1} x_{i}\right\rangle\left(J^{\prime}, K\right)$ if $\left.J^{\prime} \geqq K\right]$. On the other hand, if $J \leqq K$, we have

$$
\langle 0 z\rangle \leqq\langle 0 z\rangle_{\mathbb{Z}^{3}}, \text { i.e. } \xi_{\perp}(J, K) \leqq \xi_{\text {Bulk }}(K),
$$

and therefore we get $\xi_{\perp}(J, K)=\xi_{\text {Bulk }}(K)$, for $J \leqq K$. 


\section{The High-Temperature Behaviour of Spin-Spin Correlations}

In this section we describe the main ideas behind the proofs of results (a) and (b) described in Subsect. 1.5, see (1.18) and (1.19), taking for granted some technical results of $[12,13]$.

By performing a partial resummation of the standard high-temperature expansion (see e.g. $[24,13]$ ) one can represent the spin-spin correlation function $\langle S(x) S(y)\rangle$ as a sum over walks, $\omega$, taking nearest-neighbor steps, which start at $x$ and end at $y$. More precisely,

$$
\langle S(x) S(y)\rangle=\sum_{\omega: x \rightarrow y} \prod_{b \in \omega} K(b) z_{K, J}(\omega),
$$

where $b=\langle i, j\rangle$ is a nearest-neighbor step in $\omega$, and $K(b)=J$, for $b \subset \Sigma, K(b)=K$, otherwise. Furthermore, $z_{K, J}(\omega)$ is a positive weight on the set of walks from $x$ to $y$ with the properties that correlations between distant pieces of $\omega$ are very small, and $z_{K, J}(\omega) \rightarrow 1$, as $\max (K, J) \rightarrow 0$. For explicit representations of $z_{K, J}(\omega)$, see $[24,13]$. We now choose $x=0$ and $y=(r, 0, \ldots, 0)$.

The techniques developed in $[18,13]$ permit one to show that if either $K \ll J \ll 1$, or $J \leqq K \ll 1$ then (5.1) behaves like

$$
\text { const } r^{-\delta} \exp (-r / \xi), \text { as } r \rightarrow \infty,
$$

for some $\xi=\xi(K, J)>0$ and some $\delta$ which may be calculated as follows. Define a function $G(r)$ by

$$
G(r)=\sum_{\tilde{\omega}: 0 \rightarrow(r, 0, \ldots, 0)} \prod_{b \in \tilde{\omega}} K(b),
$$

where $\tilde{\omega}$ is an arbitrary directed walk from 0 to $(r, 0, \ldots, 0)$ without back-tracking, and $K(b)$ is as above. One then shows that $G(r)$ behaves like

$$
\text { const }^{\prime} r^{-\delta^{\prime}} \exp \left(-r / \xi^{\prime}\right), \text { as } r \rightarrow \infty,
$$

for some $\xi^{\prime}=\xi^{\prime}(K, J)>0$, with $\xi^{\prime}(K, J) / \xi(K, J) \rightarrow 1$, as $\max (K, J) \rightarrow 0$, and for some $\delta^{\prime}$. The point is now that, under the hypotheses specified above,

$$
\delta^{\prime}=\delta \text {. }
$$

The details of a proof of (5.5) are quite lengthy and technical, but it should be possible to adapt the methods of $[18,13]$ to provide them. The analysis of the behaviour of (5.3) in the two extreme situations $K \ll J \ll 1$ and $J \leqq K \ll 1$ is contained in the literature; see e.g. [11], or Sect. 3 of [18]. When $K \ll J \ll 1$, the easier case, the sum on the right-hand side of (5.3) is dominated by walks, $\tilde{\omega}$, which are contained in the surface layer, $\Sigma$, except for rare and short excursions into the bulk whose contribution can be estimated by a simple Mayer expansion for a "gas of excitations," as in $[13,18]$. Hence the power correction, $r^{-\delta^{\prime}}$, to the exponential decay of $G(r)$ is an ordinary Ornstein-Zernike correction for the generating function of directed walks, $\tilde{\omega}$, without back-tracking from 0 to $(r, 0, \ldots, 0)$ which are entirely contained in $\Sigma=\mathbb{Z}^{d-1}$. This is well-known to be $r^{-\frac{d-2}{2}}$, i.e.

$$
\delta=\delta^{\prime}=\frac{d-2}{2} .
$$

This is what has been claimed in (1.18). 
If, however, $J \leqq K \ll 1$, then the sum on the right-hand side of (5.3) is dominated by walks, $\tilde{\omega}$, contained in $\left\{j \in \mathbb{Z}^{d}: j^{d}>0\right\}$, where $j^{d}$ is the $d^{\text {th }}$ component of $j$, which have only rare and short encounters with $\Sigma$. Now, all $d-1$ components of the projection, $\hat{\omega}$, of $\tilde{\omega}$ onto a plane, $\pi$, perpendicular to the 1 -axis are simple random walks indexed by an integer time which is the 1 -coordinate of each site on $\tilde{\omega}$. The $d$-component, $\hat{\omega}^{d}$, of $\hat{\omega}$ is a simple random walk subject to the constraint

$$
\hat{\omega}^{d}(n) \geqq 0, \text { for all } n=0,1, \ldots, r .
$$

The other components, $\hat{\omega}^{\alpha}, \alpha=2, \ldots, d-1$, are simple random walks on $\mathbb{Z}$. Hence

$$
G(r)=\exp (-r / \xi) \prod_{\alpha=2}^{d} \operatorname{Prob}\left\{\hat{\omega}^{\alpha}: \hat{\omega}^{\alpha}(r)=0 \mid \hat{\omega}^{\alpha}(0)=0\right\},
$$

where, for $\alpha=2, \ldots, d-1$,

while

$$
\operatorname{Prob}\left\{\hat{\omega}^{\alpha}: \hat{\omega}^{\alpha}(r)=0 \mid \hat{\omega}^{\alpha}(0)=0\right\} \underset{r \rightarrow \infty}{\sim} r^{-1 / 2},
$$

$$
\operatorname{Prob}\left\{\hat{\omega}^{d}: \hat{\omega}^{d}(r)=0 \mid \hat{\omega}^{d}(0)=0\right\} \underset{r \rightarrow \infty}{\sim} r^{-3 / 2} .
$$

For the easy proofs, see e.g. [11]. In conclusion,

i.e.

$$
\begin{gathered}
G(r) \underset{r \rightarrow \infty}{\sim} r^{-\frac{d-2}{2} r^{-3 / 2}} \exp \left(-r / \xi^{\prime}\right)=r^{-\frac{d+1}{2}} \exp \left(-r / \xi^{\prime}\right), \\
\delta=\delta^{\prime}=\frac{d+1}{2} .
\end{gathered}
$$

This is precisely what is claimed in (1.19).

The arguments outlined here represent a complete proof of (1.18) and (1.19), assuming (5.5). The details of the proof of (5.5) which is based on an analysis of (5.1) and of the weights $z_{K, J}(\omega)$ appearing therein are rather tedious, but are sufficiently close to the arguments in $[10,12,13,18]$ that we feel we may omit a detailed presentation.

\section{References}

1. Mermin, N.D., Wagner, H.: Absence of ferromagnetism or antiferromagnetism in one- or twodimensional isotropic Heisenberg models. Phys. Rev. Lett. 17, 1133-1136 (1966)

2. Goldstone, J.: Field theories with "superconductor" solutions. Nuovo Cimento 19, 154-164 (1961);

Mermin, N.D.: Absence of ordering in certain classical systems. J. Math. Phys. 8, 1061-1064 (1967)

3. Fröhlich, J., Pfister, C.-E.: On the absence of spontaneous symmetry breaking and of crystalline ordering in two-dimensional systems. Commun. Math. Phys. 81, 277-298 (1981), Absence of crystalline odering in two dimensions (comments). 104, 697-700 (1986)

4. Pfister, C.-E.: On the symmetry of the Gibbs states in two dimensional lattice systems. Commun. Math. Phys. 79, 181-188 (1981)

5. Lipowsky, R.: Critical surface phenomena at first order bulk transitions. Phys. Rev. Lett. 49, 1575-1578 (1982); and refs. given there 
6. Fröhlich, J., Spencer, T.: Massless phases and symmetry restoration in abelian gauge theories and spin systems. Commun. Math. Phys. 83, 411-454 (1982)

7. Fröhlich, J., Simon, B., Spencer, T.: Infrared bounds, phase transitions, and continuous symmetry breaking. Commun. Math. Phys. 50, 79-95 (1976)

8. Simon, B.: Correlation inequalities and the decay of correlations in ferromagnets. Commun. Math. Phys. 77, 111-126 (1980)

9. Glimm, J., Jaffe, A.: Quantum physics. A functional integral point of view. Berlin, Heidelberg, New York: Springer 1981; and refs. given there

10. Abraham, D.: Solvable model with a roughening transition for a planar Ising ferromagnet. Phys. Rev. Lett. 44, 1165-1168 (1980)

11. Fisher, M.E.: Walks, walls, wetting, and melting. J. Stat. Phys. 34, 667-729 (1984)

12. Gallavotti, G.: The phase separation line in the two-dimensional Ising model. Commun. Math. Phys. 27, 103-136 (1972)

Bricmont, J., Lebowitz, J.L., Pfister, C.-E.: J. Stat. Phys. 26, 313-332 (1981)

13. Bricmont, J., Fröhlich, J.: Statistical mechanical methods in particle structure analysis of lattice field theories. II. Scalar and surface models. Commun. Math. Phys. 98, 553-578 (1985)

14. Camp, W.J., Fisher, M.E.: Behavior of two-point correlation functions at high temperatures. Phys. Rev. Lett. 26, 73-77 (1971), Behavior of two-point correlation functions near and on a phase boundary. 26, 565-568 (1971)

15. McBryan, O., Spencer, T.: On the decay of correlations in $S O(n)$-symmetric ferromagnets. Commun. Math. Phys. 53, 299-302 (1977)

16. Fröhlich, J., Spencer, T.: The Kosterlitz-Thouless transition in two-dimensional abelian spin systems and the Coulomb gas. Commun. Math. Phys. 81, 527-602 (1981)

17. Ginibre, J.: General formulation of Griffiths' inequalities. Commun. Math. Phys. 16, 310-328 (1970)

18. Bricmont, J., Fröhlich, J.: Statistical mechanical methods in particle structure analysis of lattice field theories. I. General theory. Nucl. Phys. B 251 [FS 13], 517-552 (1985)

19. Aizenman, M., Simon, B.: Local Ward identities and the decay of correlations in ferromagnets. Commun. Math. Phys. 77, 137-144 (1980)

20. Dunlop, F.: Correlation inequalities for multicomponent rotators. Commun. Math. Phys. 49, 247-256 (1976);

Kunz, H., Pfister, C.-E., Vuillermot, P.A.: Inequalities for some classical spin vector models. J. Phys. A 9, 1673-1683 (1976)

21. Dunlop, F., Newman, C.: Multicomponent field theories and classical rotators. Commun. Math. Phys. 44, 223-235 (1975)

22. Gruber, C., Kunz, H.: General properties of polymer systems. Commun. Math. Phys. 22, 133-161 (1971)

Cammarota, C.: Decay of correlations for infinite range interactions in unbounded spin systems. Commun. Math. Phys. 85, 517-528 (1982)

Brydges, D.C.: A short course on cluster expansions. Lectures given at the 1984 Les Houches Summer School, to appear in the proceedings. Osterwalder, K., Stora, R. (eds.)

23. Lebowitz, J.L., Monroe, J.L.: Bounds on the correlations and analyticity properties of ferromagnetic Ising spin systems. Commun. Math. Phys. 28, 313-321 (1972);

Bricmont, J.: The gaussian inequality for multicomponent rotators. J. Stat. Phys. 17, 289-300 (1977)

24. Brydges, D., Fröhlich, J., Spencer, T.: The random walk representation of classical spin systems and correlation inequalities. Commun. Math. Phys. 83, 123-150 (1982)

25. Fröhlich, J., Spencer, T.: In: Scaling and self-similarity in physics. Fröhlich, J. (ed.). Progress in Physics, Vol. 7. Boston, Basel, Stuttgart: Birkhäuser 1983

Communicated by A. Jaffe

Received June 19, 1986 